\title{
Effects of a dynamic balance training protocol on podalic support in older women. Pilot Study
}

\author{
Giuseppe Battaglia ${ }^{1,4}$, Marianna Bellafiore ${ }^{1,2,4}$, Antonino Bianco ${ }^{1,2,4}$, Antonio Paoli ${ }^{3}$ and \\ Antonio Palma ${ }^{1,2,4}$ \\ 1School of Sport and Exercise Sciences, University of Palermo, 2Department of Sports Science (DISMOT), \\ University of Palermo, ${ }^{3}$ Department of Experimental Medicine, University of Padova, ${ }^{4}$ Regional Sport \\ School of Sicily CONI (Olympic National Italian Committee), Palermo, Italy
}

ABSTRACT. Background and aims: The foot provides the only direct contact with supporting surfaces and therefore plays an important role in all postural tasks. Changes in the musculoskeletal and neurological characteristics of the foot with advancing age can alter plantar loading patterns and postural balance. Several studies have reported that exercise training improves postural performance in elderly individuals. The aim of our study was to investigate the effectiveness of a dynamic balance training protocol performed for 5 weeks on the support surface, percentage distribution of load in both feet, and body balance performance in healthy elderly women. Methods: Ten subjects (68.67 \pm 5.50 yrs old; $28.17 \pm 3.35$ BMI) were evaluated with a monopodalic performance test and baropodometric analyses before and after the training period. Results: We found a significant improvement in balance unipedal performance times on left and right foot by $20.18 \%$ and $26.23 \%$ respectively $(\mathrm{p}<0.05)$. The support surface of the right foot significantly increased in response to the training protocol and, in particular, in both forefoot and rearfoot regions $(\mathrm{p}<0.05)$. In addition, before the training period, load distribution on the left foot was greater than on the right one; equal load redistribution was measured on both feet in response to exercise (p>0.05). Conclusions: The increased support surface and equal redistribution of body weight on both feet obtained in response to our training protocol may be postural adaptations sufficient to improve static balance in elderly women.

(Aging Clin Exp Res 2010; 22: 406-411)

๑2010, Editrice Kurtis

\section{INTRODUCTION}

Multiple sensory modes are involved in the organization and control of human erect posture, in addition to the central postural program. Many studies have focused on the specific function of vestibular, visual or muscular sensory information $(1,2)$ in body balance control; however, little is known about the role of podalic support. The foot provides the only direct contact with the supporting surface and therefore plays an important role in all postural tasks. In particular, changes to the musculoskeletal and neurological characteristics of the foot associated with advancing age may potentially alter plantar loading patterns (3). It is known that foot problems affect up to $80 \%$ of older people (4) and that they are associated with impaired mobility and ability to perform common household tasks (5). Altered function of foot load distribution (3) and increased postural sway (6) have been observed to influence body balance negatively in elderly people. Indeed, individuals who have sustained multiple falls demonstrate greater postural sway than age-matched peers $(6,7)$. In particular, women have a higher risk of falls than men (810). Butler et al. found that older women performed significantly worse than older men in balance-related tests (11). Wolfson et al. (1994) found that elderly women swayed and lost their balance more than men when the surface was sway-referenced while vision was compromised. Elderly women also showed impairment of balance when simultaneously deprived of visual and somatosensory inputs or during backward destabilization. In particular, these authors explained the greater frequency of falling in women because of limited postural control in comparison with that of men in conditions stressing balance (10). For this reason, it would be interesting to study specific interventions in order to improve balance control in wom-

Key words: Body balance, exercise, foot, podalic support.

Correspondence: Giuseppe Battaglia, PhD, Faculty of Sports and Exercise Sciences, University of Palermo, Via Maggiore Toselli 87/B, 90143 Palermo, Italy.

E-mail: giuseppebattaglia@unipa.it

Received June 30, 2009; accepted in revised form November 20, 2009.

First published ahead of print December 1st, 2009 as DOI: 10.3275/6713 
en. It is known that physical activity is reduced with increasing age (12) and sedentary life represents a risk factor for decline in postural control (13). Several studies have reported that exercise training aimed at developing endurance, muscle strength, balance, mobility, flexibility and sensory abilities, separately or in combination, all improve postural performance in elderly individuals (1416). In particular, Rogers et al. showed an improvement in postural sway in elderly women after ten weeks of a training program including stretching, strengthening and balance exercises (14). Ledin et al. found improved equilibrium in elderly people in response to regular balance training exercises performed for 9 weeks (15). Similarly, Judge et al. showed a higher improvement in static balance of sedentary subjects exercised by a program including resistance training, walking and postural exercises, than a program including only postural control exercises (16). However, other authors did not find any improvement in body balance in response to exercise training in older adults $(17,18)$, and a worsening in postural control after a low-volume strength training program was even observed by Bellew et al. (19).

Little is known about the influence of exercise training on podalic support in order to improve body balance in older people. The main aim of our study was to investigate the effects of a short dynamic balance training protocol on the support surface and load distribution in healthy and active elderly women. We also examined whether changes in these baropodometric parameters affected body balance by performing a test of monopodalic orthostatic posture.

\section{METHOD}

\section{Subjects}

Participants were recruited from a senior centre in Palermo. Twenty-one subjects, 18 women and 3 men accepted taking part in the project, but only women were recruited. All women were given a questionnaire regarding their personal data, health status, time devoted to physical activity, and history of falls in the past year. After a starting interview and screening, 13 women were regarded suitable for our study, because the others did not match inclusion criteria such as being over 60 , availability to attend $80 \%$ of the training period, and suitability for practicing physical activity as attested by a medical certificate. However, only 10 women correctly followed the experimental program (three subjects did not attend $80 \%$ of the training period, for personal reasons). The women in our study were $68.67 \pm 5.50$ years old, with $63.74 \pm 7.83 \mathrm{~kg}$ weight, $1.50 \pm 0.03 \mathrm{~m}$ height, and $28.17 \pm 3.35$ BMI. All subjects were autonomous and capable of self-care and normal household tasks; none had fallen in the past year. All subjects gave their informed consent prior to participation in this study, which was approved by the local institutional ethics committee.

\section{Exercise training protocol}

In the literature, as little is known about the effects of specific training protocols of dynamic equilibrium on static postural control in elderly people, we planned a dynamic balance training protocol mainly characterized by balance exercises, to be performed during walking. These exercises were selected in order to stimulate the vestibular, visual, muscular and podalic components of the balance control system.

The training program was performed for 5 consecutive weeks and consisted of 2 sessions/week for the first 3 weeks and 3 sessions/week for the last 2 weeks. Every session included three phases: warm-up (15 minutes, min), training period (20 $\mathrm{min}$ ) and cool-down (10 min). Each subject performed the training program wearing special fitness socks (AKKUA, Brescia, Italy) in order to ensure maximum grip of feet on floor and to protect subjects from accidental falls. In the first week of the training protocol, subjects familiarized themselves with the circuit with a single lap per training session. In the subsequent weeks, participants completed two laps of the training circuit per session.

In the warm-up phase, participants executed a standard sequence of exercises, including 2 sets of: 3 clockwise (CC) and anti-clockwise (ACC) circumductions of the neck; 5 forward (FC) and backward (BC) circumductions of the shoulders; 5 CC and ACC of pelvis; $5 \mathrm{CC}$ and ACC of feet. In addition, we inserted 1-2 min of free-walking every 2 sets of joint mobility exercises.

In the training phase, participants performed circuit training with their hands on their hips in order to exclude their upper limbs during postural training exercises.

In particular, in the first three weeks circuit training included 20 exercises, of which 17 (exercises 1-11, 13-16, $18,20)$ for stress dynamic balance and $3(12,17,19)$ for static balance. The exercises were organized in a standard sequence, including: 1) forward slalom walking with step length corresponding to foot length (SLCFL); 2) body translation by lateral walking to the right; 3) forward walking (FW), alternating the feet among lines with knees up; 4) one clockwise body translation by lateral walking; 5) one anticlockwise body translation by lateral walking; 6) FW with SLCFL; 7) backward walking (BW) with SLCFL; 8) FW with SLCFL; 9) climbing one step (90 cm length, $40 \mathrm{~cm}$ width, $14 \mathrm{~cm}$ thickness) and FW with SLCFL; 10) descending one down step and FW with SLCFL; 11) climbing one step and FW with SLCFL; 12) descending one step, and standing for 10 seconds with a block of wood $(28 \mathrm{~cm}$ length, $25.5 \mathrm{~cm}$ width, $1.5 \mathrm{~cm}$ thickness, $872 \mathrm{~g}$ weight) on the head $(\mathrm{BOH})$; 13) FW on the mat (175 cm length, $70 \mathrm{~cm}$ width, $2.5 \mathrm{~cm}$ thickness) with SLCFL and $\mathrm{BOH}$; 14) $\mathrm{BW}$ on the mat with SLCFL without BOH; 15) FW on the mat with closed eyes (EC) and SLCFL; 16) FW with open eyes and SLCFL; 17) getting into monopodalic posture on right foot for 10 seconds; 18) FW with SLCFL; 19) getting into monopodalic posture on left foot for 10 seconds; 20) FW with SLCFL. 
Training intensity increased in the fourth and fifth weeks by the modification of several exercises and addition of new ones. In particular, the complexity of exercises 3 and 15 were increased by $\mathrm{BOH}$. In addition, we inserted several new exercises, such as standing for 10 seconds with $\mathrm{BOH}$ after exercises 2 and 3; BW on mat with SLCFL-EC-TOH and FW on mat with EC-SLCFL after exercise 15 performed with $\mathrm{BOH}$.

The cool-down phase included free walking, free body movement, deep breaths and isometric contractions of both arms for 5 seconds in several postures.

\section{Monopodalic test against time}

The monopodalic test against time (MTAT) consisted of standing in a monopodalic orthostatic posture from 0 to 30 seconds. It was performed with both feet with hands on hips, while subjects stared at a wall 60 centimetres $(\mathrm{cm})$ away, three times respectively. This posture during the test was different from that during exercise training. During monopodalic exercises of circuit training, subjects had hands on hips, wore anti-slip socks, did not stare at a point on a wall, and stood in monopodalic orthostatic posture for only 10 seconds.

All the times of the MTAT trials were recorded by manual chronometer (Pro Touch, CH-Ostermundigen, Bern), but only the best time of three records was considered for both MTAT on left and right feet.

\section{Baropodometric examination}

All subjects performed an electronic baropodometric test in static bipedal conditions without footwear. We used a module-platform electronic baropodometer (Physical Support, Rome, Italy) with a $120-\mathrm{cm}$ long and $40-\mathrm{cm}$ wide barosensitive platform. The board contains 4800 platinum electronic sensors covered by an alveolar rubber captor, which sends pressure information from each foot to a computer. During the test, subjects stood on the platform in an orthostatic position for 10 seconds, as in the study of Ripani et al. (20). The support surfaces of both feet and the percentage distribution of load in rearfoot and forefoot regions were evaluated. Data were acquired before and after the training protocol and processed by dedicated software (Physical Gait Software ${ }^{\mathrm{TM}}$, Rome, Italy).

\section{Statistical analysis}

All data were compared by Student's paired t-test before and after the training period. Statistical significance was set of $p<0.05$.

\section{RESULTS}

Evaluation of performance of monopodalic orthostatic posture

In MTAT, subjects showed an improvement in their ability to maintain their balance on both left and right feet after training (Fig. 1). They significantly increased their

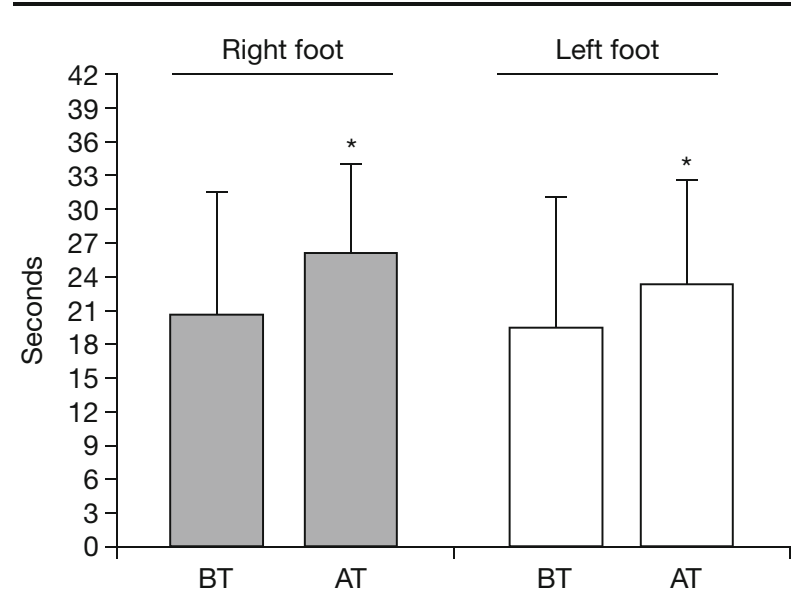

Fig. 1 - Subjects' ability to maintain balance on left and right foot before and after training period. ${ }^{*} \mathrm{p}<0.05$ after training (AT) vs before training $(B T)$.

skill at standing in a monopodalic orthostatic posture on left [before training: $19.40 \pm 11.65$ (s) vs after training: $23.31 \pm 9.25(\mathrm{~s})$ ] and right feet [before training: $20.61 \pm 10.76$ (s) vs after training: $26.02 \pm 7.96$ (s)], respectively by $20.18 \%(p<0.05)$ and $26.23 \%(p<0.05)$.

Analysis of support surfaces of left and right feet

The support surfaces (SS) of the left foot did not show any significant difference in response to training $\left(118.00 \pm 11.78\right.$ us $\left.118.10 \pm 16.20 \mathrm{~cm}^{2} ; p>0.05\right)$. Instead, the SS of the right foot increased significantly after training (122.10 \pm 16.36 us $\left.130.50 \pm 18.22 \mathrm{~cm}^{2} ; p<0.05\right)$ (Fig. 2),

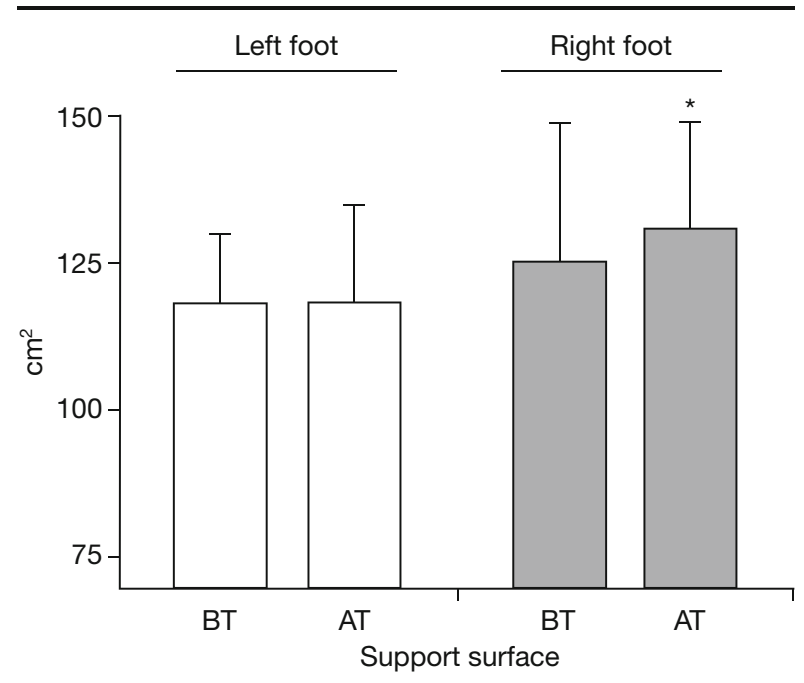

Fig. 2 - Evaluation of support surfaces of left and right feet before and after training. ${ }^{*} \mathrm{p}<0.05$ after training $(A T)$ vs before training $(B T)$. 


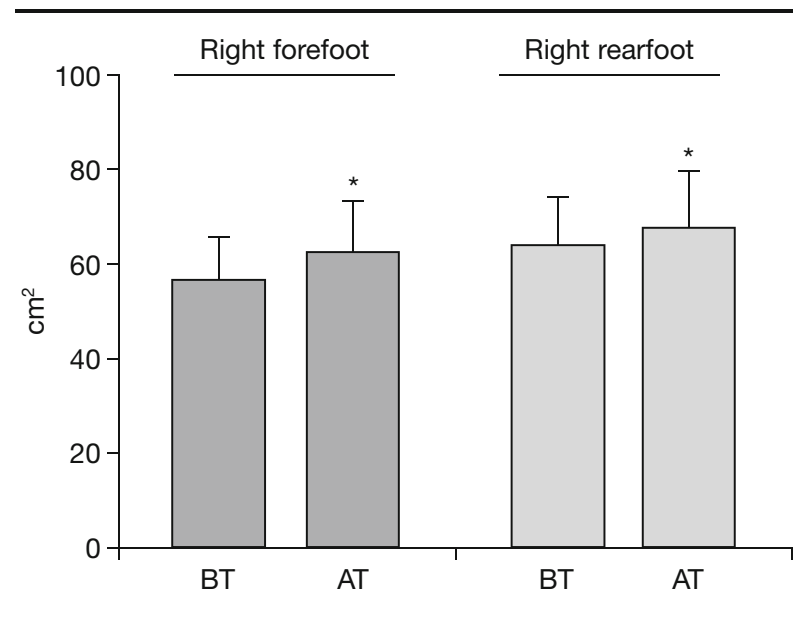

Fig. 3 - Analysis of forefoot and rearfoot surfaces of right foot before and after training program. ${ }^{*} \mathrm{p}<0.05$ after training $(A T)$ vs before training $(B T)$.

and was related to significant modifications $(p<0.05)$ in forefoot and rearfoot surfaces, which increased by $10.19 \%$ and $5.61 \%$ respectively after training (Fig. 3 ).

Percentage distribution of load on left and right feet

Before training, subjects had unequal percentage distributions of load (PDL) between left and right feet. In contrast, after training, they had equal redistributions of load between feet, although this modification was not statistically significant. In particular, total PDL on the left foot decreased $(51.30 \pm 3.71$ us $49.60 \pm 3.98 \% ; p>0.05)$ and total PDL on the right foot increased $(48.70 \pm 3.71$ us $50.40 \pm 3.98 \% ; p>0.05)$. Moreover, participants did not show any significant difference in the PDL on the forefoot and rearfoot of either foot in response to training (left forefoot: $24.40 \pm 4.09$ us $24.10 \pm 2.85 \%$; left rearfoot: $26.90 \pm 4.28$ us $25.60 \pm 2.99 \%$; right forefoot: $19.50 \pm 3.34$ vs $20.90 \pm 2.33 \%$; right rearfoot: $29.20 \pm 2.62$ us $29.50 \pm 4.43 \% ; p>0.05)$.

\section{DISCUSSION}

Our training protocol caused an increase in the total support surface, due significantly to the enhanced area in both forefoot and rearfoot of the right foot. In particular, this increase was higher in the forefoot than the rearfoot. Tanaka et al. found greater forefoot area in young subjects compared with elderly individuals, and postulated that a smaller support than that of young people was associated with poor balance (21). Therefore, the enhanced support surface observed in our study suggests the positive effect of our training protocol in maintaining standing balance. In addition, the dynamic balance exercises in our protocol, such as forward and backward walking, with step length corresponding to foot length on ground, step or mat, may have trained the ability to maintain the body's center of gravity within its base of support and induced adaptations of the balance control system in elderly subjects. It is known that one way of revealing postural control is to reduce the base of support of the center of gravity (14).

Age-related anatomical and physiological changes in foot bones and ligament structures affect the load distribution of the human body on the feet during gait (22). In our study, elderly women had a greater load distribution on the left foot than on the right before training but equal redistribution of body weight on both feet in response to exercise. Therefore, our dynamic balance protocol appears to influence both sides of the body symmetrically.

In order to examine whether the changes in support surface and load distribution induced by our training program affected body balance, we evaluated static balance performance and found that, after five weeks of training, older women improved their monopodalic performance times on both feet respectively by $20.18 \%$ (left) and $26.23 \%$ (right). In particular, average times of performance ranged from 20.61 to $26.02 \mathrm{~s}$ and 19.40 to $23.31 \mathrm{~s}$ on right and left feet, respectively.

Hurvitz et al. showed that older ambulatory outpatients with a history of falling had times of $<30$ seconds during unipedal stance tests, while those with a low risk of falling had times of $\geq 30$ seconds, suggesting that the unipedal stance test from 0 to 30 seconds is an indicator of fall risk among older outpatients (23). According to that study, our subjects appeared to be liable to falls before and after training. However, our training protocol was efficient in increasing the times of balance performance and therefore may protect older women from risk of falls. Sakamoto et al. showed that unipedal standing balance exercise was effective in preventing falls among clinically defined high-risk elderly individuals (24). In agreement with the present results, similar exercises in our training protocol may contribute to improved postural control in older people. Although different performance conditions were used during exercises in the training program and monopodalic test (MTAT), monopodalic exercises may have improved the response of the balance control system in the monopodalic test. However, the number of other exercises, such as walking on the mat with open or closed eyes, or with a wooden block on the head, may have stimulated concerted interaction among the neuromusculoskeletal, proprioceptive, vestibular and visual components of the postural system and thus improved body balance. Hue et al. showed that tailored three-month physical activity programs improved the postural stability of older people in the foam floor condition, with open and closed eyes (25). However, Lord et al. reported that the use of mats with open eyes did not lead to improvements in the proprioceptive system, because sub- 
jects were compelled to rely on visual and vestibular sensory and motor systems to maintain stability when reliable proprioceptive information from feet and ankles was altered under the particular stance required by a foam surface (26).

In our training protocol, several exercises were performed with closed eyes, and a block of wood on the head may have stressed the proprioceptive function, which is known to be related to the closed-loop control mechanisms of the balance system (27). However, Collins et al. showed that, during quiet stance, the postural system uses open-loop control mechanisms over short-term intervals and closed-loop control ones in long-term intervals (27). Open-loop mechanisms operate without sensory feedback and may correspond to descending commands, which set the steady-state activity levels of the postural muscles. Because the exercises in our protocol had shortterm intervals, we suggest that an adaptation of open-loop control mechanisms took place.

The significant increase in the support surface of the right foot may be due to the dominant limb phenomenon and contribute to greater improvement in balance unipedal performance on the right foot rather than the left one. Improved balance unipedal performance on the left foot may be due to interpodalic contralateral transfer processes of motor learning (28). The equal redistribution of body weight on both feet induced by our training protocol may be important in improving balance in elderly women. In the future, it may be interesting to study the relationship between baropodometric parameters and functional dominance in older people.

\section{CONCLUSIONS}

Our dynamic balance training protocol, characterized by a gradual increase in the workload, appears to be practicable, safe and repeatable by older people. The increased support surface and equal redistribution of body weight on both feet obtained in response to training may lead to postural adaptations sufficient for improving static balance in elderly women. The application of this protocol in senior centres may favor a reduction in fall risk and promote socialization among elderly persons. This study also adds to our knowledge about ways in which the age-related decline in the neuromusculo-skeletal system can be contrasted. It would be interesting to insert this protocol in an annual program of functional movement re-education for older people with balance disorders. For example, it could be motivating to perform this training protocol together with a specific strength protocol, in order to test the effects on balance control of these two different but complementary training exercises.

In the future, we intend to extend this pilot study to a larger scale and to investigate its effects in young people, middle-aged adults and older people.

\section{ACKNOWLEDGEMENTS}

The authors are grateful to CIVES senior centre of Palermo (Italy), Ignazio Accomando and Donatella Bonafede for their collaboration.

\section{REFERENCES}

1. Wernick-Robinson M, Krebs DE, Giorgetti MM. Functional reach: does it really measure dynamic balance? Arch Phys Med Rehabil 1999; 80: 262-9.

2. Choy NL, Brauer, S, Nitz J. Changes in postural stability in women aged 20 to 80 years. J Gerontol A Biol Sci Med Sci 2003; 58: 525-30

3. Scott G, Menz HB, Newcombe L. Age-related differences in foot structure and function. Gait Posture 2007; 26: 68-75.

4. Harvey I, Frankel S, Marks R, Shalom D, Morgan M. Foot morbidity and exposure to chiropody: Population based study. BMJ 1997; 315: 1054-5

5. Benvenuti F, Ferrucci L, Guralnik JM, Gangemi S, Baroni A. Foot pain and disability in elderly persons: an epidemiologic survey. $\mathrm{J}$ Am Geriatr Soc 1995; 43: 479-84.

6. Era P, Heikkinen E. Postural sway during standing and unexpected disturbance of balance in random samples of men of different ages. J Gerontol 1985; 40: 287-95.

7. Crilly RG, Delaquerrière-Richardson L, Roth JH, Vandervoort AA, Hayes KC, Mackenzie RA. Postural stability and Colles' fracture. Age Ageing 1987; 16: 133-8.

8. Tinetti ME, Speechley M, Ginter SF. Risk factors for falls among elderly persons living in the community. N Engl J Med 1988; 319: 1701-7.

9. Sattin RW, Lambert Huber DA, DeVito CA et al. The incidence of fall injury events among the elderly in a defined population. Am J Epidemiol 1990; 131: 1028-37.

10. Wolfson L, Whipple R, Derby CA, Amerman P, Nashner L. Gender differences in the balance of healthy elderly as demonstrated by dynamic posturography. J Gerontol 1994; 49: M160-7.

11. Butler AA, Menant JC, Tiedemann AC, Lord SR. Age and gender differences in seven tests of functional mobility. J Neuroeng Rehabil 2009; 30: 6-31.

12. Aijö M, Heikkinen E, Schroll M, Stehen B. Physical activity and mortality of 75-year-old people in three Nordic localities: a fiveyear follow-up. Aging Clin Exp Res 2002; 14 (Suppl 3): 83-9.

13. Unsworth J, Mode A. Preventing falls in older people: risk factors and primary prevention through physical activity. $\mathrm{Br} \mathrm{J}$ Community Nurs 2003; 8: 214-20.

14. Rogers ME, Fernandez JE, Bohlken RM. Training to reduce postural sway and increase functional reach in the elderly. $J$ Occup Rehabil 2001; 11: 291-8.

15. Ledin T, Kronhed AC, Möller C, Möller M, Odkvist LM, Olsson B. Effects of balance training in elderly evaluated by clinical tests and dynamic posturography. J Vestib Res 19901991; 1 : 129-38

16. Judge JO, Lindsey C, Underwood M, Winsemius D. Balance improvements in older women: effects of exercise training. Phys Ther 1993; 73: 254-62.

17. Crilly RG, Willems DA, Trenholm KJ, Hayes KC, DelaquerrièreRichardson LF. Effect of exercise on postural sway in the elderly. Gerontology 1989; 35: 137-43.

18. Topp R, Mikesky A, Dayhoff NE, Holt W. Effect of resistance training on strength, postural control, and gait velocity among older adults. Clin Nurs Res 1996; 5: 407-27. 
19. Bellew JW, Yates JW, Gater DR. The initial effects of low-volume strength training on balance in untrained older men and women. J Strength Cond Res 2003; 17: 121-8.

20. Ripani M, Ciccarelli A, Morini S, Ricciardi G, Michielon G. Evaluation of foot support in rugby players: a baropodometric analysis. Sport Sci Health 2006; 1: 104-8.

21. Tanaka T, Takeda H, Izumi T, Ino S, Ifukube T. Effects on the location of the centre of gravity and the foot pressure contribution to standing balance associated with ageing. Ergonomics 1999; 42: 997-1010.

22. Rodgers MM. Dynamic foot biomechanics. J Orthop Sports Phys Ther 1995; 21: 306-16.

23. Hurvitz EA, Richardson JK, Werner RA, Ruhl AM, Dixon MR. Unipedal stance testing as an indicator of fall risk among older outpatients. Arch Phys Med Rehabil 2000; 81: 587-91.

24. Sakamoto K, Nakamura T, Hagino $\mathrm{H}$ et al. Committee on
Osteoporosis of The Japanese Orthopaedic Association. Effects of unipedal standing balance exercise on the prevention of falls and hip fracture among clinically defined high-risk elderly individuals: a randomized controlled trial. J Orthop Sci 2006; 11 : 467-72.

25. Hue OA, Seynnes O, Ledrole D, Colson SS, Bernard PL. Effects of a physical activity program on postural stability in older people. Aging Clin Exp Res 2004; 16: 356-62.

26. Lord SR, Menz HB. Visual contributions to postural stability in older adults. Gerontology 2000; 46: 306-10.

27. Collins JJ, De Luca CJ, Burrows A, Lipsitz LA. Age-related changes in open-loop and closed-loop postural control mechanisms. Exp Brain Res 1995; 104: 480-92.

28. Prokop T, Berger W, Zijlstra W, Dietz V. Adaptational and learning processes during human split-belt locomotion: interaction between central mechanisms and afferent input. Exp Brain Res 1995; 106: 449-56. 